\title{
Terpsinoë musica Ehrenberg (Bacillariophyceae), primer registro del género para Colombia
}

\author{
Terpsinoë musica Ehrenberg (Bacillariophyceae), first record of the genus for \\ Colombia
}

\author{
Mario F. Medina-Tombé ${ }^{*}$, Amelia A. Vouilloud ${ }^{2}$, Silvia E. Sala ${ }^{2}$
}

\begin{abstract}
Resumen
En este trabajo se presenta el primer registro de la especie Terpsinoë musica y la primera cita del género para Colombia. Los especímenes de $T$. musica se estudiaron mediante microscopía óptica y electrónica de barrido, se realizó la descripción morfológica y se tomaron los datos morfométricos. Esta alga fue encontrada en muestras de perifiton recolectadas en sistemas lóticos de las regiones biogeográficas de la Orinoquía y el Caribe, asociada a Eichhornia crassipes y Lenna minor, en cuerpos de agua levemente alcalinos, con conductividades eléctricas moderadas y bajos valores de caudal. Se realizó una comparación con otras poblaciones de la especie registradas en América, Europa y África, mencionando aspectos relacionados con sus requerimientos ecológicos y distribución geográfica. El material tipo de la especie no ha sido analizado con técnicas modernas de estudio. Teniendo en cuenta la gran variabilidad morfológica entre distintas poblaciones de la especie y la diversidad de ambientes en los que se registró, se plantea si estamos frente a un taxón con amplia variabilidad morfológica y gran distribución geográfica o, por el contrario, si se trata de un complejo de especies con requerimientos ecológicos y distribuciones más restringidas.
\end{abstract}

Palabras claves: diatomea, perifiton, tropical, primera cita

\begin{abstract}
The first record for Colombia of the species Terpsinoë musica and the first citation for the genus for Colombia. The specimens of $T$. musica were studied using optic and scanning microscopy. The morphological description was made and morphometric data were taken. This alga was found in periphyton samples which were collected in lotic ecosystems in the biogeographic regions Orinoquia and Caribbean, associated with Eichhornia crassipes and Lenna minor in alkaline ecosystems with moderate electrical conductivities and low values of water level. A comparison with other populations of the species registered for America, Europe and Africa was made, mentioning aspects related to their ecological requirements and geographic distribution. The type material of the species has not been analyzed using modern techniques. It is suggested we are facing a taxon with high morphological variation and wide geographical distribution, taking on count the huge morphological variation between different populations of the species and the diversity of environments where it was registered, or on the contrary, it is about a complex of species with more limited ecological requirements and distributions.
\end{abstract}

Keywords: diatom, periphyton, tropical, first citation

\footnotetext{
1. Grupo de Investigación en Limnología Básica y Experimental y Biología y Taxonomía Marina, Universidad de Antioquia, Medellín, Colombia.

2. División Ficología Dr. Sebastián Guarrera, Facultad de Ciencias Naturales y Museo, Paseo del Bosque s/n. 1900 La Plata, Argentina.

* Autor de correspondencia: <mfernando621@yahoo.com>
} 


\section{INTRODUCCIÓN}

Terpsinoë Ehrenberg 1843, es un género de diatomea céntrica perteneciente a la familia Biddulphiaceae (Bacillariophyta), morfológicamente caracterizado por tener valvas elípticas, con márgenes ondulados, barras transversales prominentes, un rimoportula de posición central y un pseudocelo en cada ápice (Round et al. 1990, Sterrenburg 1994). Es un género con un número reducido de especies, en general citado para ambientes continentales tropicales a subtropicales, aunque también se registra en ambientes marino costeros (Carmona-Jiménez et al. 2017, Round et al. 1990, Tuji 2018).

Terpsinoë musica Ehrenberg (1843) es la especie tipo del género y debe su nombre a que las barras transversales rectas se hallan engrosadas hacia el centro, concediéndole un aspecto semejante a las notas musicales cuando los frústulos se observan en vista conectival. Las células vivas están unidas entre sí por un mucílago secretado por los pseudocelos formando colonias en zig-zag (Boyer 1900, Carmona-Jiménez et al. 2017, Sterrenburg 1994). En su descripción original, Ehrenberg mencionó que fue hallada en materiales colectados en el municipio de Atotonilco El Grande (México), sin incluir referencia alguna respecto al sitio de recolección. Posteriormente se registró en diversos sistemas continentales y marinos costeros de América (Carmona-Jiménez et al. 2017, Luttenton et al. 1986, Sterrenburg 1994, Vouilloud 2003), Europa (Peragallo y Peragallo 1897, Van Heurck 1899), África (Baudrimont 1973, ElAwamri et al. 2003), e islas del Pacífico (Ehrenberg 1843, Navarro y Lobban 2009). Por registrarse en ambientes tan diversos es considerada una especie eurihalina (Baudrimont 1973, Navarro y Lobban 2009, Van Heurck 1899), que prolifera con mayor frecuencia en aguas duras con temperaturas cálidas (Sterrenburg 1994). Puede colonizar una gran diversidad de sustratos, ya que se ha encontrado sobre rocas (ElAwamri et al. 2003), en raíces de árboles sumergidos y briófitas acuáticas (Luttenton et al. 1986), posada en otras algas filamentosas como Cladophora (ElAwamri et al. 2007) y Bostrychia (Azevedo 1999) y también formando parte del perifiton de los ríos (Carmona-Jiménez et al. 2017).
La recopilación de los trabajos taxonómicos realizada por Montoya et al. (2013) y la revisión de trabajos posteriores publicados para Colombia, mostraron que no existe ningún registro previo de alguna especie de este género para el país.

\section{MATERIALES Y MÉTODOS}

Este trabajo forma parte de una investigación que abarcó 18 sistemas lóticos de las zonas biogeográficas de los Andes (en el departamento de Santander), Caribe (en el departamento de Magdalena), Orinoquía (en el departamento de Arauca) y Pacífico Colombiano (en el departamento de Nariño) (figura Ш). El material analizado en este estudio se recolectó en dos sistemas lóticos de la región de la Orinoquía (Río Lipa confluencia con el arroyo Primores y Río Lipa aguas arriba; estos sitios se ubicaron a $134 \mathrm{~m}$ s. n. m. y geográficamente entre los $6^{\circ} 47^{\prime} 6,7^{\prime \prime} \mathrm{N}$ y $71^{\circ} 1^{\prime} 34^{\prime \prime} \mathrm{W}$, y $6^{\circ} 49^{\prime} 33,2^{\prime \prime} \mathrm{N}$ y $71^{\circ} 2^{\prime} 16,1^{\prime \prime} \mathrm{W}$, respectivamente) y en dos del Caribe (en el municipio de Ciénaga, departamento de Magdalena, en los arroyos Canta Rana (910 m s. n. m., $11^{\circ} 0^{\prime} 13,9^{\prime \prime}$ $\mathrm{N}$ y $\left.74^{\circ} 3^{\prime} 46,8^{\prime \prime} \mathrm{W}\right)$ y El Padre (928 m s. n. m., $10^{\circ} 58^{\prime} 36,1^{\prime \prime} \mathrm{N}$ y $74^{\circ} 4^{\prime} 22,7^{\prime \prime} \mathrm{W}$ ) (figura 四).

En campo, las muestras del perifiton se recolectaron sobre sustratos rocoso y macrófitas (10 sustratos en cada sitio), cepillando una superficie de $90 \mathrm{~cm}^{2}$ por sitio. Cada muestra se fijó con Lugol (al 10\%) y 6 a 8 gotas de formalina. Adicionalmente se midió la elevación sobre el nivel del mar con un GPS (Garmin etrex 20x), el caudal por medio del método de flotación (ISO 1998), la temperatura con un termómetro de mercurio, el pH con un pH-metro Martini (pH56 $0,01 \mathrm{pH}$ ), la conductividad eléctrica con un medidor de conductividad Martini (Conductividad 0-3.999 $\mu \mathrm{S} / \mathrm{cm}$ ) y el oxígeno disuelto por medio de titulación con Winkler (Merck) (tabla 1).

En el laboratorio las muestras se trataron con agentes oxidantes para eliminar la materia orgánica siguiendo el método descrito en CENT/TC 230 (2002). Para observar las muestras en el microscopio óptico (MO) el material tratado se montó en Naphrax ${ }^{\circledR}$, y para el microscopio electrónico de barrido (MEB), se montó sobre tacos de vidrio y se recubrió con una capa 
fina de oro paladio en un Jeol JFC-1100 (fine coat ion sputter). Las observaciones se realizaron con un microscopio 500 LM Leica DM y en un microscopio electrónico de barrido Jeol JSM-6490LV MEB de la Universidad de Antioquia, Colombia. Las muestras fueron depositadas en la Colección de la División Ficología del Museo de La Plata (LPC). En la tabla 1 se alistan las muestras empleadas y los números de la colección.

La terminología utilizada para la descripción de los taxones es la sugerida en Barber y Harworth (1981). Para el ordenamiento sistemático se siguió a Cox (2015).

\section{RESULTADOS}

Valvas elípticas con márgenes triondulados; las ondulaciones o ensanchamientos tienen aproximadamente la misma proporción entre sí (figura \2A-E)

$\begin{array}{ll}\text { Clase } & \text { Mediophyceae } \\ \text { Subclase } & \text { Biddulphiophycidae } \\ \text { Familia } & \text { Biddulphiaceae } \\ \text { Género } & \text { Terpsinoë } \\ \text { Especie } & \text { Terpsinoë musica Ehrenberg } \\ & \text { 1843, p.425, Pl. 3, figs. IV-1 - } \\ & \text { VII- 30. figura } \square(\mathrm{A}-\mathrm{I}), 3 \text { (A-E). }\end{array}$

a veces con una ligera reducción a la altura de las penúltimas barras transversales. Ápices capitados a rostrados con un pseudocelo formado por finos poroides organizados en hileras irregulares (figura [2I). La superficie externa de la valva está cubierta por areolas fácilmente visibles al MO (figura $2 \mathrm{~F}-\mathrm{F}$ ), dispuestas irregularmente en el centro y formando líneas irregulares más o menos longitudinales hacia los extremos (figura $2 \mathrm{G}$ ); al MEB se distinguen como rugosidades que se disponen de forma irregular por toda la valva (figura $2 \mathrm{ZH}-\mathrm{I}$ ). La valva tiene un rimoportula posicionada excéntricamente en el lóbulo

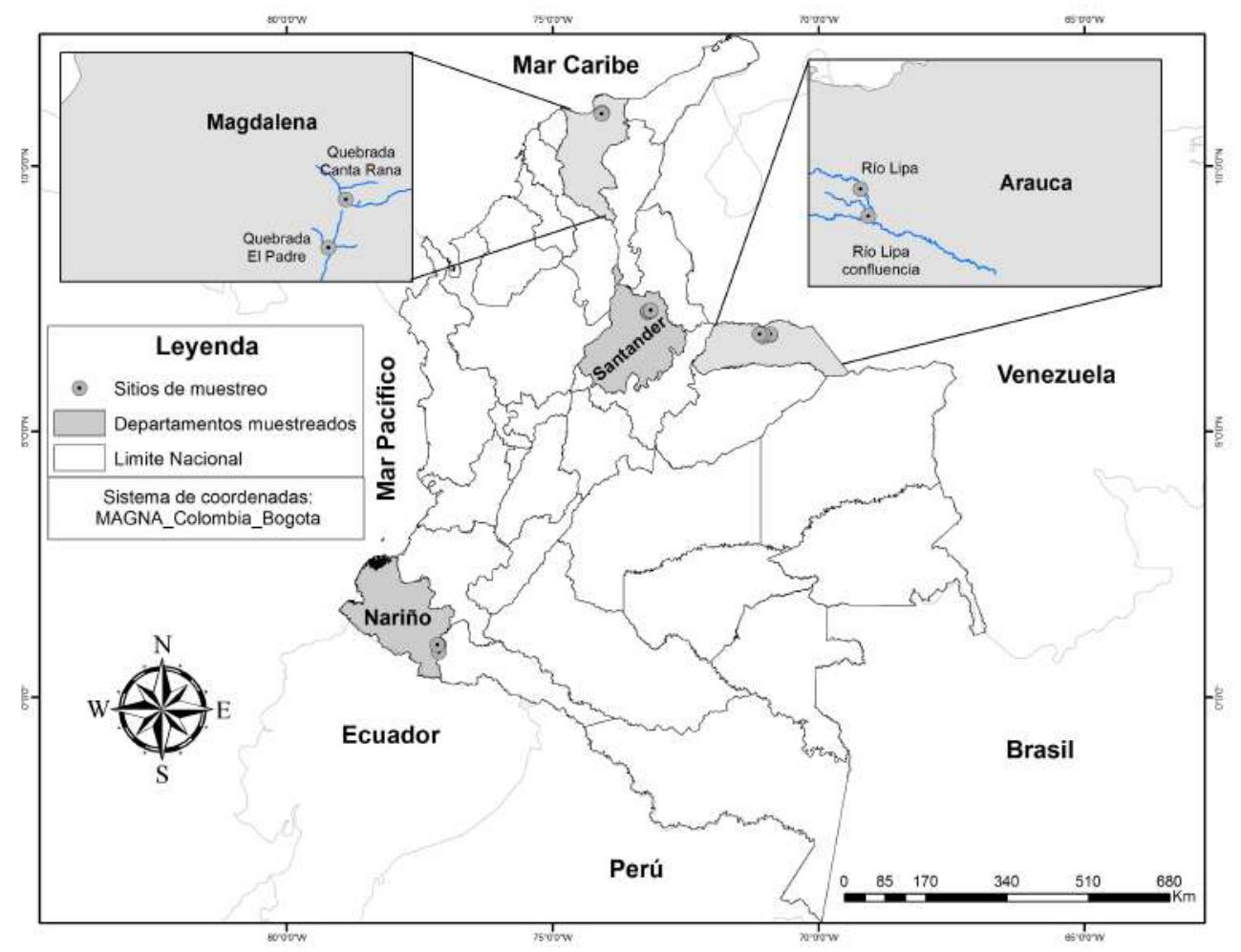

Figura 1. Ubicación geográfica de los sitios de muestreo en las cuatro regiones biogeográficas estudiadas. Regiones donde se registró Terpsinoë musica: Región Caribe: departamento del Magdalena y Región Orinoquía: departamento de Arauca. 
central (figura $2 \mathrm{~F}$ y $\mathrm{G}$ ). Cada valva presenta seis barras transversales que se ordenan transapicalmente entre las porciones ensanchadas y los pseudocelos (figura 叉A-D, 2G).

Frústulos rectangulares en vista conectival (figura BA y B). Las barras transversales se observan en esta vista ocupando hasta $3 / 4$ de la altura del manto, con los extremos engrosados de modo que en conjunto presentan aspecto de nota musical. Las barras transversales que se encuentran en las porciones ensanchadas son más largas que las que se encuentran cerca de los pseudocelos (figura BA y B). Manto valvar alto, ornamentado con areolas irregularmente dispuestas (figura $\mathbf{B C}$ ). Cingulum compuesto por pocas bandas cerradas (figura $\mathbf{B C}$ ); valvocópula con poroides pequeños dispuestos irregularmente. El borde de la pars interior presenta fimbrias (figura $\mathrm{BD}$ y E).

Datos morfométricos ( $\mathrm{n}=30)$ : Eje apical: 105-170 $\mu \mathrm{m}(\bar{x}=120,2 \mu \mathrm{m} ; \mathrm{CV}=8,30)$; eje transapical (centro): $26,3-38,5 \mu \mathrm{m}(\bar{x}=33,3 \mu \mathrm{m} ; \mathrm{CV}=15,26)$; L/A: 2,9-4,2; eje prevalvar: 43,5-106,4 $\mu \mathrm{m}(\bar{x}=62,9$ $\mu \mathrm{m} ; \mathrm{CV}=42,99)$; Altura del manto: $17,1-25,5 \mu \mathrm{m}$ $(\bar{x}=20,9 \mu \mathrm{m} ; \mathrm{CV}=12,36)$; areolas: $7-9$ en $10 \mu \mathrm{m}$. en el ejemplar fotografiado al MEB, la densidad de los poroides del pseudocelo fue de 66-70 en $10 \mu \mathrm{m}$ y la densidad de poroides en la valvocópula fue de 15-18 en $10 \mu \mathrm{m}$.

En este estudio esta especie se encontró asociada a comunidades propias del epifiton (sobre Eichhornia crassipes y Lenna minor) del río Lipa, perteneciente a la cuenca del río Orinoco, y del epiliton en los arroyos Canta Rana y El Padre, ubicados en la región Caribe. Como se detalla en la tabla 1, en los sitios mencionados las aguas tienden a la alcalinidad con valores de $\mathrm{pH}$ entre 7,4 y 8,1 , valores de conductividad eléctrica medianamente elevados (entre 60 y 140 $\mu \mathrm{S} / \mathrm{cm}$ ) y bajos valores de caudal (entre $0,15-1,67 \mathrm{~m}^{3}$ $\left.\operatorname{seg}^{-1}\right)$ (tabla 1$)$.

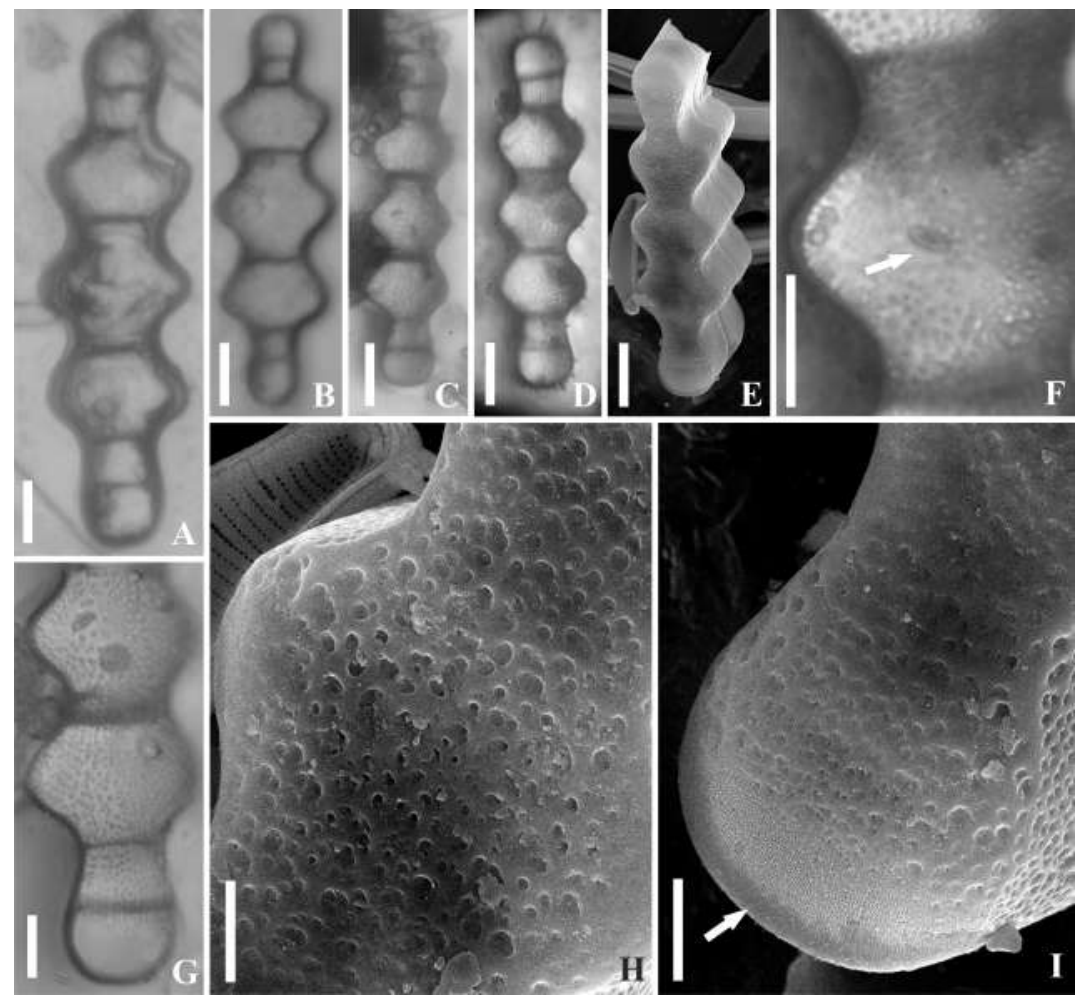

Figura 2. Terpsinoë musica. Especímenes en vista valvar. A-D, F-G. MO. E, H-I. MEB. A-E. Vistas generales. F. Detalle del rimoportula (flecha). F-H. Detalle de las areolas en la superficie valvar. I. Detalle del pseudocelo (flecha). Escalas: A-E: $20 \mu \mathrm{m}$; F: $10 \mu \mathrm{m}$; G-I: $5 \mu \mathrm{m}$. 

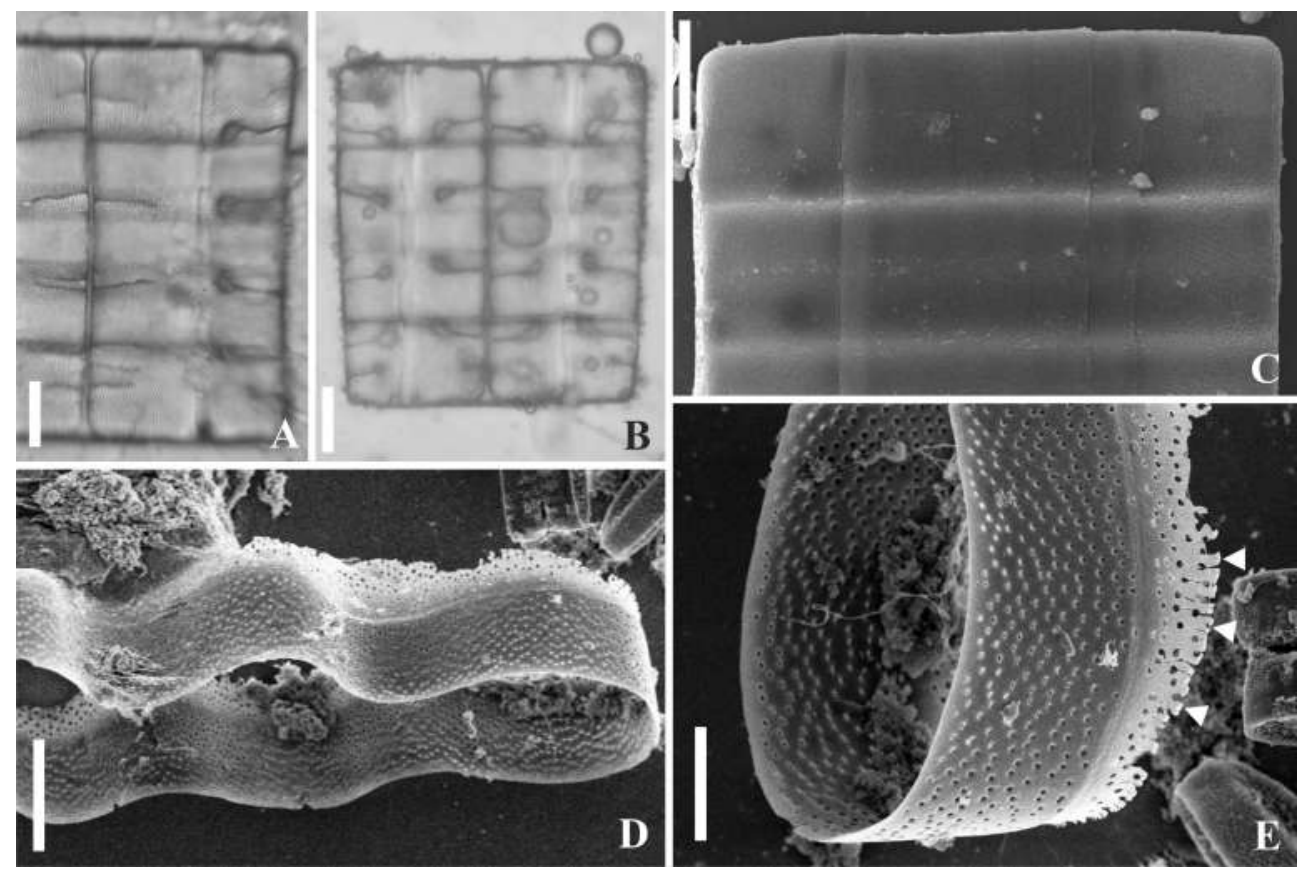

Figura 3. Terpsinoë musica. A-B. MO. Especímenes en vista conectival. C-E. MEB. A-B. Detalle de las barras transversales. C. Detalle del manto valvar y del cigulum. D-E. Detalle de la valvocópula. Escalas: A-C: 20 $\mu \mathrm{m}$; D-E: $5 \mu \mathrm{m}$.

\section{DISCUSIÓN}

La comparación de los ejemplares de T. musica hallados en Colombia respecto a otras poblaciones de $T$. musica registradas en otros sitios, muestra que estas especies tienen una amplia variabilidad morfológica, particularmente en la longitud del eje apical y del eje pervalvar (tabla 2). Coinciden en aquellos aspectos que definen la especie tales como contorno de la valva, presencia y posición del rimoportula, caracteres de las barras transapicales y pseudocelos. Una diferencia destacable es la disposición de las aréolas en la superficie valvar; aparentemente aleatoria o irregular en el centro (Boyer 1900, Carmona-Jiménez et al. 2017, El-Awamri et al. 2003, Frenguelli y Cordini 1937, Luttenton et al. 1986 y nuestro material) o radiales desde un punto central y luego paralelas/longitudinales hacia los extremos (Baudrimont 1973). Por otra parte, Carmona-Jiménez et al. (2017) documentaron algunos especímenes que presentaron pequeñas espinas en el margen valvar y más de un rimoportula.

Respecto a las características ecológicas, en general se la considera una especie de ambientes continentales salobres (aunque pueden ocurrir también en aguas dulces o marinas costeras) con una distribución tropical a subtropical (Baudrimont 1973, CarmonaJiménez et al. 2017, El-Awamri et al. 2003, Luttenton et al. 1986, Round et al. 1990, Sterrenburg 1994). Según El-Awamri et al. (2003), la especie se desarrolla mejor en aguas duras, ligeramente alcalinas y salobres, con una conductividad eléctrica relativamente alta y temperaturas moderadamente altas. Por otra parte, Baudrimont (1973) la registró en aguas bicarbonatadas, sulfatadas y clorosulfatadas y la clasificó como una especie $\beta$ mésohalobe y alcalófila, que crece sobre filamentos de Cyanophyceae y Stigeoclonium. Carmona-Jiménez et al. (2017) la observaron en aguas neutras a ligeramente alcalinas (pH 7-8 unidades), con conductividades eléctricas entre medias a altas $\left(268-2640 \mu \mathrm{S} \mathrm{cm}^{-1}\right)$ y dominadas por sulfato/bicarbonato y calcio/magnesio. Asimismo, Luttenton et al. (1986) la registraron en aguas alcalinas, de conductividades moderadamente altas y altos valores de dureza. En Colombia se hallaron en el epifiton y el epiliton de sistemas lóticos en ambientes con aguas ligeramente alcalinas y de bajo caudal. 
Tabla 1. Valores de las variables físicas y químicas medidas en los sitios de muestreo de las regiones del Caribe y Orinoquía, Colombia - donde se registró Terpsinoë musica.

\begin{tabular}{|c|c|c|c|c|c|c|c|c|c|c|}
\hline LPC* & Colector & Sistema & Coordenadas & $\begin{array}{l}\text { Altitud (m } \\
\text { s. n. m.) }\end{array}$ & $\begin{array}{l}\text { Temperatura } \\
\left({ }^{\circ} \mathrm{C}\right)\end{array}$ & $\begin{array}{l}\mathrm{pH} \\
\text { (Unidades) }\end{array}$ & $\begin{array}{l}\text { Conducti- } \\
\text { vidad } \\
(\mu \mathrm{S} / \mathrm{cm})\end{array}$ & $\begin{array}{l}\text { Oxígeno } \\
\text { disuelto } \\
\text { (mg/L) }\end{array}$ & $\begin{array}{l}\text { Caudal } \\
\left(\mathrm{m}^{3} / \mathrm{seg}^{1}\right)\end{array}$ & Sustrato \\
\hline 5572 & \multirow{4}{*}{$\begin{array}{l}\text { Mario } \\
\text { Medina }\end{array}$} & $\begin{array}{l}\text { Río Lipa } \\
\text { confluencia } \\
\text { arroyo } \\
\text { Primores }\end{array}$ & $\begin{array}{l}6^{\circ} 47^{\prime} 6,7^{\prime \prime} N \\
71^{\circ} 1^{\prime} 34^{\prime \prime} \mathrm{W}\end{array}$ & 134 & 31,4 & 8,04 & 140 & 5,5 & 0,76 & Epifiton \\
\hline 5571 & & $\begin{array}{l}\text { Río Lipa aguas } \\
\text { arriba }\end{array}$ & $\begin{array}{llll}6^{\circ} & 49^{\prime} & 33,2^{\prime \prime} \mathrm{N} \\
71^{\circ} & 2^{\prime} & 16,1^{\prime \prime} \mathrm{W}\end{array}$ & 134 & 29,5 & 7,4 & 100 & 5,5 & 0,46 & Epifiton \\
\hline 5568 & & $\begin{array}{l}\text { Arroyo Canta } \\
\text { Rana }\end{array}$ & $\begin{array}{ll}11^{\circ} 0^{\prime} 13,9^{\prime \prime} \mathrm{N} \\
74^{\circ} 3^{\prime} 46,8^{\prime \prime} \mathrm{W}\end{array}$ & 910 & 20,3 & 8,1 & 63 & 8 & 1,67 & Epiliton \\
\hline 5570 & & $\begin{array}{l}\text { Arroyo El } \\
\text { Padre }\end{array}$ & $\begin{array}{l}10^{\circ} 58^{\prime} 36,1^{\prime \prime} \mathrm{N} \\
74^{\circ} 4^{\prime} 22,7^{\prime \prime} \mathrm{W}\end{array}$ & 928 & 18,3 & 7,9 & 92 & 7,5 & 0,15 & Epiliton \\
\hline
\end{tabular}

* División Ficología del Museo de La Plata 
Tabla 2. Comparación de los datos morfológicos y morfométricos de poblaciones de Terpsinoë musica registradas en Colombia y en otras regiones del mundo.

\begin{tabular}{|c|c|c|c|c|c|c|c|c|c|c|c|}
\hline Referencia & Localidad & $\begin{array}{l}\text { Eje } \\
\text { apical } \\
(\mu \mathrm{m})\end{array}$ & $\begin{array}{l}\text { Eje } \\
\text { trans- } \\
\text { apical } \\
(\mu \mathrm{m})\end{array}$ & L/A & $\begin{array}{l}\text { Profundi- } \\
\text { dad del } \\
\text { manto } \\
(\mu \mathrm{m})\end{array}$ & $\begin{array}{l}\text { Eje per- } \\
\text { valvar } \\
(\mu \mathrm{m})\end{array}$ & $\begin{array}{l}\text { Núm. } \\
\text { barras } \\
\text { trans- } \\
\text { versa- } \\
\text { les }\end{array}$ & $\begin{array}{l}\text { Densidad } \\
\text { de areolas } \\
\text { en } \\
\text { superficie } \\
\text { valvar en } \\
10 \mu \mathrm{m}\end{array}$ & Rimoportula & Pseudocelo & Espinas \\
\hline $\begin{array}{l}\text { Carmona } \\
\text { Jiménez } \\
\text { et al. } \\
\text { (2017) }\end{array}$ & $\begin{array}{l}\text { Tropical } \\
\text { streams } \\
\text { from } \\
\text { Mexico }\end{array}$ & $\begin{array}{l}80,2- \\
135,6\end{array}$ & $\begin{array}{l}33,6- \\
50\end{array}$ & - & $16-24 \dagger$ & $64-122$ & $2-6$ & 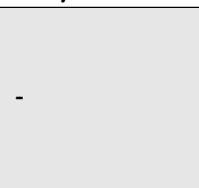 & $\begin{array}{l}\text { 1-(2-3), } \\
\text { apertura } \\
\text { interna en } \\
\text { forma de } S\end{array}$ & $\begin{array}{l}\text { Poroides } \\
\text { aleato- } \\
\text { rimente } \\
\text { distribui- } \\
\text { das }\end{array}$ & $\begin{array}{l}\text { Pequeñas } \\
\text { espinas } \\
\text { margina- } \\
\text { les }\end{array}$ \\
\hline $\begin{array}{l}\text { El- } \\
\text { Awamri } \\
\text { et al. } \\
(2003)\end{array}$ & $\begin{array}{l}\text { El Cairo, } \\
\text { Egipto }\end{array}$ & $\begin{array}{l}111- \\
140\end{array}$ & $35-49$ & 3 & $12-17$ & $64-105$ & 6 & $12-14$ & $\begin{array}{l}1 \text {, apertura } \\
\text { interna en } \\
\text { forma de } \mathrm{S}\end{array}$ & $\begin{array}{l}\text { Poroides } \\
\text { irregu- } \\
\text { larmente } \\
\text { dispuestas }\end{array}$ & No \\
\hline $\begin{array}{l}\text { Wu } \\
(2013)\end{array}$ & - & $56-133$ & $32-44$ & $\begin{array}{l}1,6- \\
3,7\end{array}$ & $21-27$ & $52-99$ & $2-6$ & - & 1 & 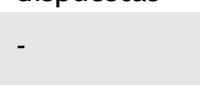 & No \\
\hline $\begin{array}{l}\text { Baudrimont } \\
\text { (1973) }\end{array}$ & Argelia & $40-200$ & - & - & - & $35-40$ & 6 & 10 & 1 & - & No \\
\hline $\begin{array}{l}\text { Luttenton } \\
\text { et al. } \\
(1986)\end{array}$ & $\begin{array}{l}\text { Oklahoma, } \\
\text { EE.UU }\end{array}$ & $49-116$ & $37-43$ & - & - & - & $2-6$ & - & 1 & - & No \\
\hline $\begin{array}{l}\text { Navarro } \\
\text { y Lobban } \\
(2009)\end{array}$ & Guam & $\begin{array}{l}100- \\
110\end{array}$ & $85-95$ & - & - & - & - & $8-10$ & - & - & No \\
\hline $\begin{array}{l}\text { Este } \\
\text { estudio }\end{array}$ & $\begin{array}{l}\text { Regiones } \\
\text { de } \\
\text { Orinoquía } \\
\text { y El } \\
\text { Caribe }\end{array}$ & $\begin{array}{l}105- \\
170\end{array}$ & $25-30$ & $\begin{array}{l}4,2- \\
5,6\end{array}$ & $14-26$ & $68-88$ & 6 & $8-10$ & 1 & $\begin{array}{l}66-70^{* *} \\
\text { poroides } \\
\text { dispuestos } \\
\text { en hileras } \\
\text { irregulares }\end{array}$ & No \\
\hline
\end{tabular}

$\dagger$ En la descripción de T. musica, Carmona. Jimenez et al. (2017), expresan que la profundidad del frústulo es de 64,4-125 $\mu$ m, casi el mismo rango que para la medida del eje pervalvar; consideramos que se trata de un error y por tando las medidas incluidas en la presenta tabla son las resultantes de medir los ejemplares de las fotos publicadas.

** dato tomado a partir de un único ejemplar. 
Si bien la mayoría de las citas se refieren, en efecto, a localidades tropicales a subtropicales, la especie también ha sido citada en ambientes templados de agua dulce, como es el caso del río Delaware (Boyer 1900) y el lago Michigan en USA (Wujek y Welling 1981), en arroyos de la provincia de Buenos Aires, Argentina (Vouilloud 2003) y en sedimentos cuaternarios de la llanura pampeana, Argentina (Frenguelli y Cordini 1937, Frenguelli 1945).

En la descripción original de T. musica, Ehrenberg (1843) destacó la presencia de las estructuras con forma de notas musicales como característica de la especie y la ilustró con dibujos de dos ejemplares observados en vista conectival. No aportó ningún detalle de la vista valvar ni parámetros morfométricos, por lo que la descripción resulta insuficiente al momento de establecer comparaciones con poblaciones registradas en otros estudios. El material tipo de T. musica no ha sido estudiado posteriormente por ningún otro autor. Wu (2013), basado en la descripción original de T. musica y considerando la gran variabilidad morfológica entre diferentes poblaciones de la especie, determinó que es posible que estemos frente a un complejo de especies (flock), y en este sentido la localidad tipo es un dato importante al momento de analizar su taxonomía. Tuji (2018), empleando técnicas moleculares, comparó la población tipo de T. muninensis Tuji con una población de $T$. musica de Texas (sitio cercano a la localidad tipo de T. musica); el autor interpretó que las diferencias halladas en las secuencias apoyan la justificación de la descripción de su nuevo taxón, resaltando la importancia de considerar la distribución geográfica como factor relevante en el tratamiento taxonómico.

Para resolver esta cuestión es imprescindible, en primer lugar, analizar el material tipo de T. musica con técnicas modernas de análisis morfológico, a fin de establecer su identidad y así comparar la morfología fina de las distintas poblaciones y determinar si estamos frente a un taxón con una gran variabilidad morfológica y amplia distribución geográfica o, por el contrario, si se trata de un complejo de especies con requerimientos ecológicos y distribuciones más restringidas.

\section{AGRADECIMIENTOS}

Esta investigación contó con el apoyo financiero del Instituto de Investigación de Recursos Biológicos Alexander von Humboldt (Proyecto Planeación ambiental Ndeg 5211478 (IAvH12-067), el Grupo de Investigación LimnoBase y Biotamar de la Universidad de Antioquia, Colombia.

\section{CONFLICTO DE INTERESES}

Los autores declaran no tener conflictos de intereses.

\section{REFERENCIAS}

Azevedo A. 1999. Composição florística de diatomáceas (Bacillariophyta) epífitas em duas espécies de Bostrychia Montage (Rhodophyta). Insula Florianópolis, 28: 101-148. https://periodicos.ufsc.br/index.php/ insula/article/view/21988/19944

Barber H, Haworth E. 1981. A guide to the morphology of the diatom frustule: with a key to the British freshwater genera. Ambleside (UK): Freshwater Biological Association.

Baudrimont R. 1973. Precisions sur lecologie, en Algerie, de Terpsinoë musica Her. (Bacillariophycées Anaulacées). Bulletin de la Société linéenne de Bordeaux, 3(1): 21-24. https://archive.org/details/bulletindel3519731975soci/ page/n27

Boyer C. 1900. The biddulphoid forms of North American Diatomaceae. Proceedings of the Academy of Natural Sciences of Philadelphia, 52: 685-748. https://www.jstor. org/stable/4062683?seq=1\#metadata_info_tab_contents

Carmona-Jimenez J, Beltrán Y, Salinas-Camarillo V. 2017. Morphological and environmental characterization of Terpsinoё musica (Biddulphiaceae, Bacillariophyceae) in tropical streams from Mexico. Diatom Research, 32(2): 185-193. https://doi.org/10.1080/0269249X.2017. 1.3352:38

CEN/TC 230. 2002. Water quality-guidance standard for the routine sampling and pretreatment of benthic diatoms from rivers. Brussels (Belgium): European Standard. CEN/TC 230 prEN 13946: Committee on European Normalization.

Cox E. 2015. Diatoms. En: Frey W editor. Syllabus of Plant Families - A. Engler's Syllabus der Pflanzenfamilien Part 2/1: Photoautotrophic eukaryotic Algae. Glaucocystophyta, Cryptophyta, Dinophyta/Dinozoa, Haptophyta, Heterokontophyta/Ochrophyta, Chlorarachniophyta/Cercozoa, Euglenophyta/Euglenozoa, Chlorophyta, Streptophyta p.p. Stuttgart: Borntraeger Science Publishers. p. 64-102.

Ehrenberg C. 1843. Verbreitung und Einfluss des mikroskopischen Lebens in Süd-und Nord-Amerika. Abhandlungen der Königlichen Akademie der 
Wissenchaften zu Berlin, 1841: 291-466. https:// diatoms.org/citations/ehrenberg_cg-1843-verbreitung_und_einfluss_des_mikroskopischen_lebens_in_su

El-Awamri A, Shaaban A, Saleh A. 2003. Morphology and ecology of the centric diatom Terpsinoë musica Ehrenberg, based on material collected from Cairo, Egypt. Egyptian Journal of Phycology, 4(2): 161-174. Www.ejmanager. com/mnstemps/167/167-1466490944.pdf

El-Awamri A, Shaaban A, Saleh A. 2007. Floristic study on benthic diatoms of the groundwater seepages at Kobri El-Kobba (Cairo, Egypt). Journal of Applied Sciences Research, 3(12): 1809-1818. http://www.aensiweb.com/ old/jasr/jasr/2007/1809-1818.pdt

Frenguelli J, Cordini I. 1937. La diatomita de Quilino (Provincia de Córdoba), su contenido y sus posibilidades de explotación. Revista del Museo de La Plata (Nueva Serie), Sección Geología, 1: 67-116.

Frenguelli J. 1945. El Platense y sus diatomeas, Las diatomeas del Platense. Revista del Museo de La Plata (Nueva Serie), Sección Paleontología, 3: 77-221.

Luttenton M, Pfiester L, Timpano P. 1986. Morphology and growth habit of Terpsinoë musica Ehr. (Bacillariophyceae). Castanea, 51(3): 172-182. http://www.jstor.org/ stable/4033384

Montoya Y, Sala S, Vouilloud A, Aguirre N, Plata Y. 2013. Lista de las diatomeas de ambientes continentales de Colombia. Biota Colombiana, 14(2): 13-78. http://www. redalyc.org/articulo.oa?id=49131094002

Navarro J, Lobban C. 2009. Freshwater and marine diatoms from the western Pacific islands of Yap and Guam, with notes on some diatoms in damselfish territories. Diatom Research, 24(1): 123-157. DOI: 10.1080/0269249X.2009.9705787

Peragallo H, Peragallo M. 1897. Diatomées marines de France et des districts maritimes voisins. J. Tempere. Atlas 24 pls. Grez-sur Loing (S.-et-M.): J. Tempère, MicrographeEditeur. https://doi.org/10.5962/bhl.title.13501

Round F, Crawford R, Mann DG. 1990. The diatoms: biology and morphology of the genera. New York: Cambridge University Press.

Sterrenburg F. 1994. Terpsinoë musica Ehrenberg (Bacillariophyceae, centrales), With emphasis on protoplast and cell division. Netherlands Journal of Aquatic Ecology, 28(1): 63-69. https://doi.org/10.1007/BF02334246

Tuji A. 2018. A new freshwater diatom, Terpsinoë muninensis sp. nov., from the Ogasawara Islands, Japan. Memoirs of the National Museum of Nature and Science, Tokyo, 52: 5-15. http://www.kahaku.go.jp/research/ publication/memoir/download/52/52_5.pdf

Van Heurck H. 1896. A treatise on the Diatomaceae. London: William Wesley Son.

Vouilloud AA. 2003. Catálogo de las diatomeas continentales y marinas de Argentina. La Plata (Argentina): Asociación Argentina de Ficología.

Wu S. 2013. Terpsinoë musica. In Diatoms of the United States. Colorado (USA): Diatoms of North America; [fecha de acceso agosto 19, 2017]. http://westerndiatoms.colorado. edu/taxa/species/terpsinoe_musica

Wujek DE, Welling ML. 1981. The occurrence of two centric diatoms new to the Great Lakes. Journal of Great
Lakes Research, 7(1): 55-56. https://doi.org/10.1016/ S0380-1330(81)72024-0 